\title{
Assimetria de Tonsilas Palatinas: Experiência de 10 Anos do Serviço de Otorrinolaringologia do Hospital de Clínicas da Universidade Federal do Paraná
}

\author{
Palatine Tonsils Asymmetry: 10 Years Experience of the Otorhinolaryngology \\ Service of the Clinical Hospital of the Federal University of Paraná
}

\author{
Annelyse Cristine Ballin*, Heloisa Nardi Koerner**, Carlos Henrique Ballin***, Rodrigo Pereira ****, \\ LauroJoão Lobo Alcântara*****, Guilberme Ribas Taques******, Marcos Mocellin*******.
}

\footnotetext{
* Médica Residente do $3^{\circ}$ Ano do Serviço de Otorrinolaringologia do HC-UFPR.

** Médica Residente do $2^{\circ}$ Ano do Serviço de Otorrinolaringologia do HC-UFPR

*** Médico Graduado pela UFPR.

***** Médico Otorrinolaringologista do Hospital Pequeno Príncipe de Curitiba.

****** Médico Otorrinolaringologista do HC-UFPR e do Hospital Pequeno Príncipe de Curitiba.

****** Médico Residente do $3^{\circ}$ Ano do Serviço de Anatomia Patológica do HC-UFPR.

******** Médico Otorrinolaringologista. Chefe do Serviço de Otorrinolaringologia do HC-UFPR
}

Insituição: Universidade Federal do Paraná.

Curitiba / PR - Brasil

Endereço para correspondência: Heloisa Nardi Koerner - Rua Buenos Aires, 600 conj. 1901- Bairro: Batel - Curitiba / PR - Brasil - CEP: $80250-070$ - Telefone: (+55 41$)$ 9996-2303 - E-mail: helo_nk@ hotmail.com

Artigo recebido em 13 de Setembro de 2010. Artigo aprovado em 10 de Dezembro de 2010

\section{RESUMO}

Introdução:

Objetivo:

Método:

Resultados:

Conclusões:

Palavias-chave:

\section{SUMMARY}

Introduction:

Objective:

Method:

Results:

Conclusion:

Keywords:
Poucos pacientes com amígdalas palatinas assimétricas apresentam linfoma, entretanto a maioria dos linfomas em amígdalas palatinas cursam com assimetria amigdaliana.

Relatar o perfil dos pacientes e das alterações histopatológicas em pacientes submetidos a adenoamigdalectomia/ amigdalectomia com amígdalas palatinas desproporcionais.

Estudo retrospectivo, baseado na análise de dados do Banco de Dados do Serviço de Anatomia Patológica e na revisão dos prontuários de pacientes submetidos a adenoamigdalectomias e amigdalectomias com assimetria de amígdalas palatinas durante o período de outubro de 1999 a outubro de 2009 no Hospital de Clínicas (HC) da Universidade Federal do Paraná (UFPR).

Foram incluídos 50 pacientes, com idade entre 3 a 53 anos, idade média de 14,05 anos. O exame anatomopatológico evidenciou 48 pacientes (96\%) com hiperplasia linfoide e 2 casos de linfoma (4\%). Estes eram homens de 40 e 53 anos com queixa de aumento unilateral da amígdala, um deles apresentava outros sintomas (astenia e emagrecimento). Ao exame físico: desproporção amigdaliana, sem envolvimento de outros órgãos ou linfonodos. Nosso estudo concorda com a literatura quanto aos linfomas serem mais comuns em homens, o paciente ser o primeiro a notificar o aumento de volume e a assimetria ser maior que 2 graus entre as amígdalas palatinas em casos de linfoma. Embora raro, mesmo na presença de disparidades, há chances da assimetria cursar com um quadro maligno. A necessidade de avaliação anatomopatológica de uma peça deve ser considerada juntamente a outros dados clínicos que sugiram um quadro maligno em vigência. tonsila palatina, tonsilite, neoplasias tonsilares, tonsilectomia.

A few patients with asymmetric palatine tonsils have lymphoma; but most lymphomas in palatine tonsils evolve with tonsillar asymmetry

To report the profile of patients and histopathological changes in patients submitted to adenotonsillectomy/ tonsillectomy with disproportional palatine tonsils.

Retrospective study based on data analysis from the Pathologic Anatomy Service Database and in the review of reports of patients submitted to adenotonsillectomy and tonsillectomy with palatine tonsils asymmetry during the period from October 1999 through October 2009 at the Clinical Hospital (HC) of the Federal University of Paraná (UFPR).

50 patients took part, aged between 3 to 53 years old, mean age of 14.05 years. The anatomopathological exam confirmed 48 patients (96\%) with lymphoid hyperplasia and 2 cases of lymphoma (4\%). These included men aged from 40 to 53 yeas with complaint of unilateral increase of the tonsil, one of whom had other symptoms (asthenia and weight loss). Upon physical examination: tonsils disproportion without involvement of other organs or lymph nodes.

Our study is according to the literature as regards the fact the lymphomas are more common in men, the patient is the first one to notice the increase in volume and the asymmetry is larger than 2 degrees between palatine tonsils in cases of lymphoma. Although it is rare, even in the presence of differences, there are chances for the asymmetry to evolve to a malignant profile. The need for anatomopathological evaluation of a part must be considered along with other clinical data that suggests a malignant profile.

palatine tonsil, tonsillitis, tonsil neoplasms, tonsillectomy. 


\section{INTRODUÇÃO}

Tonsilectomias são um dos procedimentos cirúrgicos mais realizados na otorrinolaringologia. As principais indicações para este procedimento são: tonsilites de repetição, apneia obstrutiva do sono e assimetria de tonsilas palatinas com suspeita de malignidade.

A análise histopatológica rotineira da maioria dos tecidos removidos do corpo humano é consenso geral. Em relação aos produtos de tonsilectomias, diversos estudos, com séries expressivas, demonstram que os custos não compensam a realização do exame histopatológico, já que a maioria dos casos são de hiperplasias linfoides e naqueles casos confirmados de neoplasias já havia suspeição préoperatória $(1,2,3)$.

ODepartamento de Otorrinolaringologia do Hospital de Clínicas (HC) da Universidade Federal do Paraná (UFPR) realiza, rotineiramente, a avaliação histopatológica dos produtos de tonsilectomias com suspeição de neoplasias e, obrigatoriamente, em todos os casos de assimetria de tonsilas palatinas.

Muitas vezes o paciente chega ao consultório do otorrinolaringologista preocupado por ter uma assimetria tonsilar. A conduta do otorrinolaringologista deve ser a de não atrasar e realizar o diagnóstico em um caso de câncer. Ao mesmo tempo, não se deve indicar um procedimento que requer anestesia geral e tem seus riscos próprios sem uma indicação plausível. Sendo assim, estudos relacionados ajudam o otorrinolaringologista a adotar uma conduta correta.

Diversos estudos avaliam o resultado histopatológico de todos os casos submetidos a tonsilectomias, encontrando baixa incidência de linfoma. Dolev et al. avaliaram os casos com diagnóstico de linfoma de tonsila palatina e verificaram que todos os pacientes apresentavam assimetria (6).

Sendo o principal achado clínico do linfoma de tonsilas palatinas a assimetria tonsilar (4), o exame anatomopatológico torna-se fundamental em casos de aumento unilateral do volume amigdaliano, principalmente quando há suspeição da doença.

O objetivo deste trabalho foi relatar o perfil dos pacientes tonsilas palatinas assimétricas, além das principais alterações histopatológicas encontradas em suas peças cirúrgicas de tonsilectomias. Todos os pacientes avaliados são provenientes do Ambulatório de Otorrinolaringologia do Hospital de Clínicas da UFPR no período dos últimos 10 anos.

\section{MÉTODO}

Esta pesquisa foi submetida e aprovada pelo Comitê de Ética em Pesquisa do Hospital de Clínicas da Universidade Federal do Paraná.

Trata-se de um estudo retrospectivo, baseado na análise de dados de banco do Departamento de Anatomia Patológica do HC-UFPR e na revisão dos prontuários dos pacientes submetidos a tonsilectomias com assimetria de amígdalas, durante o período de outubro de 1999 a outubro de 2009, no Hospital de Clínicas da Universidade Federal doParaná.

O banco de dados do Departamento de Anatomia Patológica do HC contempla o nome do paciente, registro do hospital, data do procedimento e tipo da peça enviada. Foram revisados todos os pacientes com a peça intitulada amígdala, tonsila palatina, tonsila e peça da cavidade oral.

No estudo foram incluídos todos os pacientes submetidos a tonsilectomia ou adenotonsilectomia associada à assimetria amigdalina.

Como critérios de exclusão foram consideradas as lesões ulceradas ou vegetantes localizadas nas tonsilas palatinas, pacientes com tonsilas simétricas e material enviado para avaliação histopatológica não localizado nas tonsilas palatinas.

As tonsilas palatinas foram classificadas de acordo com o esquema proposto por BRodsky. Considerou-se como: grau 0, tonsilas em sua loja não causando obstrução da via aérea; grau I, tonsilas levemente fora da fossa tonsilar com obstrução de uma área menor que $25 \%$ das vias aéreas; grau II, tonsilas obstruindo a via aérea entre $25 \%$ e $50 \%$; grau III, tonsilas obstruindo de $50 \%$ a $75 \%$ da via aérea; grau IV, tonsilas com obstrução maior que $75 \%$ da via aérea. A diferença de 1 grau ou mais entre as tonsilas palatinas foi considerada como tonsilas assimétricas.

Os dados coletados dos prontuários foram: idade, sexo, indicações da cirurgia, grau das amígdalas palatinas, procedimento realizado (adenotonsilectomia, tonsilectomia) e resultado do exame anatomopatológico.

Todos os pacientes foram submetidos à anestesia geral, realizando as cirurgias mediante incisão arciforme no pilar anterior e dissecção periamigdaliana a frio. As tonsilas faríngeas foram curetadas. Os pacientes foram submetidos aos procedimentos de acordo com indicações clínicas. Os produtos foram imediatamente colocados em vidros nãoestéreis com formol a 10\% e enviadas para estudo histopato- 
lógico no Departamento de Anatomia Patológica do Hospital de Clínicas da UFPR.

O material foi mantido no formol por 24 horas e, após desidratação, foi emblocado em parafina sendo realizados cortes histológicos com espessura de $5 \mathrm{~mm}$, que foram corados por Hematoxilina-Eosina e analisados por microscopia óptica. Os exames histopatológicos foram realizados pelos patologistas da Disciplina de Anatomia Patológica, seguindo padrões de uniformidade.

\section{RESULTADOS}

Foram revisados 69 prontuários de pacientes com produto cirúrgico enviado ao Departamento de Anatomia Patológica intitulado amígdala, tonsila palatina, tonsila e peça da cavidade oral, totalizando 69 pacientes. Destes, em dez casos o material enviado tinha origem fora das tonsilas palatinas, 5 pacientes não apresentavam assimetria de tonsilas palatinas descrita no prontuário e 4 apresentavam lesão ulcerada nas tonsilas palatinas, sendo excluídos dotrabalho.

Dos 50 pacientes incluídos no estudo, 23 (46\%) eram do sexo feminino e 27 (54\%) do sexo masculino. A idade dos pacientes variou de 3 a 53 anos, com uma idade média de 14,05 anos. Os pacientes menores de 18 anos foram 39 (78\%). Apenas 11 (22\%) tinham idade entre 18 e 53 anos.

Foram realizadas 31 (62\%) adenotonsilectomias e 19 (38\%) tonsilectomias. A indicação por hipertrofia de tonsilas palatinas e faríngea e infecções de repetição ocorreu em 28 casos (56\%). A indicação por hipertrofia das tonsilas palatinas e infecções de repetição contabilizou 17 casos (34\%). As hipertrofias de tonsilas faríngea e assimetria das tonsilas palatinas foram 3 casos (6\%) e apenas 2 pacientes (4\%) tinham indicação cirúrgica devido ao aumento de volume tonsilar associado à assimetria de tonsilas palatinas.

Conforme o esquema proposto por BRoDsKy, cada tonsila foi classificada e avaliado quantos graus de diferença haviam entre elas. Na avaliação da hipertrofia cada tonsila palatina foi classificada quanto ao tamanho em 4 grupos: grau I, II, III e IV, com os seguintes resultados: I : 28 (28\%); II : 20 (20\%); III: 34 (34\%); IV: 18 (18\%). Apresentaram diferença de 1 grau entre as duas tonsilas palatinas 21 pacientes (42\%), diferença de 2 graus 21 pacientes (42\%) e diferença de mais de 2 graus 8 pacientes (16\%).

Em relação aos exames anatomopatológicos de tonsilas palatinas e faríngeas, observaram-se 28 pacientes (56\%) com hiperplasia linfoide, 14 pacientes (28\%) com hiperplasia linfoide folicular, 6 pacientes (12\%) com hiperplasia linfoide e inflamação aguda supurativa focal e 2 casos de linfoma (4\%). Dentre os casos de hiperplasia linfoide e inflamação aguda supurativa focal, 2 (4\%) pacientes apresentavam pequenos grãos constituídos por colônias de Actinomyces sp. dentro das criptas das tonsilas palatinas.

Entre os pacientes com linfoma, VAA, 40 anos, masculino, apresentava queixa de aumento de volume de amígdala direita há cerca de 6 meses, negava febre, emagrecimento, sudorese noturna ou outros sintomas. Ao exame físico apresentava tonsila palatina direita grau III e esquerda grau I, sem linfonodomegalia palpável. Realizou amigdalectomia, o anatomopatológico evidenciou linfoma maligno imunoblástico. Submetido à quimioterapia por cerca de 6 meses, está em remissão completa da doença há 1 ano. Paciente JBL, 53 anos, masculino, referia aumento de amígdala direita, astenia, emagrecimento e disfagia. No exame apresentava amígdala direita grau IV e esquerda grau I, sem linfonodomegalia palpável. Submetido a amigdalectomia, o anatomopatológico mostrou linfoma maligno de baixo grau de malignidade. Realizou acompanhamento com a oncologia de outro hospital, não constando à evolução deste paciente no prontuário.

Entre os 4 pacientes com lesão ulcerada nas tonsilas palatinas, todos apresentavam odinofagia e o resultado anatomopatológico foi de carcinoma de células escamosas moderadamente diferenciado invasor.

\section{DISCUSSÃO}

Diversos estudos com casuísticas grandes demonstram que o exame anatomopatológico de rotina das tonsilectomias apresenta uma relação custo/benefício negativa, não justificando sua solicitação quando não existem outras evidências clínicas sugerindo neoplasia $(1,2)$. Tal conduta também é adotada em nosso Serviço de Otorrinolaringologia.

A assimetria tonsilaréuma das indicações cirúrgicas, porém essa indicação não deve ser ampliada para qualquer tipo de assimetria. Beaty et al. definiu em 1998 alguns fatores de risco para a malignidade tonsilar como: história prévia de câncer de cabeça e pescoço, assimetria tonsilar, lesão visível ou de consistência endurecida à palpação da tonsila, perda inexplicada de peso ou presença sintomas constitucionais sem outra justificativa e a presença de massa cervical (3).

A principal alteração histológica encontrada foi à hiperplasia linfoide, dado compatível com o encontrado na literatura mundial. Porém as tonsilas podem ser sede de neoplasias de cabeça e pescoço. Destas, 25\% serão benig- 
nas, tais como papilomas escamosos, linfangiomas e cistos epidermoides. Entre as malignas, carcinomas de células escamosas, linfomas e outros carcinomas linfoepiteliais (5).

Os linfomas compreendem várias doenças linfoproliferativas divididas em Linfoma de Hodgkin (DH) e Linfomas não-Hodgkin (LNH). Em 25 a 30\% dos casos de LNH o acometimento é extranodal, sendo este mínimo (em torno de 1\%) no Linfoma de Hodgkin. O acometimento extra-nodal da cabeça e pescoço ocorre em torno de 10$30 \%$, sendo que o anel de Waldeyer encontra-se envolvido em $60-70 \%$ desses casos $(7,8)$. Em relação a todos os sítios extra-nodais, o anel de Waldeyer encontra-se acometido em apenas 10-15\% dos casos. Já o acometimento da tonsila palatina ocorre em 80\% dos LNH que acometem o Anel de Waldeyer (9).

Neste trabalho observamos dois casos de linfoma com acometimento de tonsila palatina, demonstrando um incidência de $4 \%$ na população avaliada. Nos dois casos o linfoma apresentava-se como primário de tonsila palatina, sem envolvimento de outros órgãos ou cadeias ganglionares. Hanna et al. descrevem acometimento de linfonodos associados em 20\% dos casos (7).

Segundo Spinou et al, o linfoma de amígdala é mais comum em homens, com idade acima de 45 anos e o paciente é o primeiro a notificar o aumento tonsilar (10). No nosso estudo, os 2 casos eram do sexo masculino e os pacientes foram os primeiros a referir o aumento de volume da tonsila palatina o que corrobora a literatura, apesar de um dos casos apresentar 40 anos de idade.

Dor e ulceração em tonsilas são mais comuns em pacientes com carcinoma do que com linfoma, mas o diagnóstico só pode ser confirmado com exame histopatológico cuidadoso (7). Neste estudo, todos os pacientes com ulceração em tonsilas possuíam dor e foram diagnosticados com carcinoma de células escamosas. Por esse motivo, excluímos do estudo aqueles pacientes com ulcerações nas tonsilas palatinas.

Revisando a literatura, fica claro que a principal malignidade acometendo tonsila palatina em adultos é o carcinoma de células escamosas. Em crianças, qualquer malignidade tonsilar é muito rara, mas os linfomas compõem o diagnóstico mais provável (11). Nenhuma criança apresentou neoplasia na casuística avaliada, o que reforça a ocorrência mais comum em adultos.

Um fato importante relatado em Felix et al. é com relação ao custo de cada exame antomopatológico. O autor cita que o custo do exame para o governo é em torno de 14 reais, para uma peça apenas (4). O envio de todas as peças de tonsilas para análise seria oneroso principalmente aos hospitais públicos, que sofrem com a falta de recursos financeiros.

Ao saber que as amostras positivas para malignidade giram em torno de 0,19\% (4), que as tonsilectomias são uma das cirurgias mais realizadas no mundo e que um quadro de malignidade geralmente está associado a outros achados clínicos que podem ser evidenciados na anamnese e exame físico, o estudo anatomopatológico de todas as peças cirúrgicas de tonsilectomias não se torna eficiente, nem compensatório. Tal estudo deve ser reservado para casos de suspeição, sendo que a assimetria amigdaliana, é, sem dúvida, o principal deles.

\section{CONCLUSÃO}

Embora poucos pacientes com assimetria de tonsilas palatinas apresentem linfoma, a maioria dos pacientes com linfoma de amígdalas apresenta assimetria tonsilar, o que não nos permite acreditar que a assimetria de tonsilas palatinas seja um sinal benigno. A análise anatomopatológica de todas as peças de tonsilectomia nãoé necessária, porém a avaliação das tonsilas suspeitas, principalmente se o paciente tiver outros sintomas associados que sugiram uma doença maligna torna-se primordial.

\section{REFERÊNCIAS BIBLIOGRÁFICAS}

1. Erdag TK, Ecevit MC, Guneri EA, Dogan E, IkizAO, Sutay S. Pathologic evaluation of routine tonsillectomy and adenoidectomy specimens in the pediatric population: is it really necessary? Int J Pediatr Otorhinolaryngol. 2005, 69(10):1321-5.

2. Ikram M, Khan MA, Ahmed M, Siddiqui T, Mian MY. The histopathology of routine tonsillectomy specimens: results of a study and review of literature. Ear Nose Throat J. 2000, 79(11):880-2.

3. Beaty MM, Funk GF, Karnell LH et al. Risk Factors for malignancy in adult tonsils. Head Neck. 1998, 20:339-403.

4. Felix et al. Avaliação dautilidade do exame histopatológico como rotina em tonsilectomias. Rev Bras Otorrinolaringol. 2006, 72(2):252-5.

5. Younis RT, Hesse SV, Anand VK. Evaluation of the utility and cost-effectiveness of obtaining histopathologic diagnosis on all routine tonsillectomy specimens. Laryngoscope. 2001, 111(12):2166-9.

6. Dolev Y, Daniel S, The presence of unilateral tonsillar enlargement in patients diagnosed with palatine tonsil 
lymphoma: Experience at a tertiary care pediatric hospital. Int J Pediatr Otorhinolaryngol. 2008, 72(1):9-12.

7. Hanna E, Wanamaker J, Adelstein, Tubbs R, Lavertu P. Extranodal lymphomas of the head and neck: a 20-year experience. Arch Otolaryngol Head Neck Surg. 1997, 123(12):1318-23.

8. Tokunaga M, Sato E. Non-Hodgkin's lymphoma in the southern perfecture in Japan: na analysis of 715 cases. Cancer. 1980, 46:1231-9.
9. EndoS, Kida A, Sawada U, Sugutani M, Furusaka T, Yamada Y. Clinical analysis of malignant lymphomas of the tonsils. Acta Otolaryngol Suppl. 1996, 523:263-6.

10. Spinou C, Kubba H, Konstantinidis I, Johnston A. Role of tonsillectomy in histology for adults with unilateral tonsillar enlargement. Br J Oral Maxillofac Surg. 2005, 43(2):144-7.

11. Smitheringale A. Lymphomas presenting in Waldeyer's Ring. J Otolaryngol. 2000, 29(3):183-5. 